\title{
Urine Drug Testing in the Treatment of Chronic Noncancer Pain in a Kentucky Private Neuroscience Practice: The Potential Effect of Medicare Benefit Changes in Kentucky
}

John W. Gilbert, MD, G. R. Wheeler, MD, G. E. Mick, DO, B. B. Storey, MD, S. L. Herder, MD, G. B. Richardson, MD, E. Watts, MD, K. Gyarteng-Dakwa, MD, B. S. Marino, MD,

C. M. Kenney III, MD, M. Siddiqi, MD, and P. G. Broughton, ARNP

From: Spine and Brain Neurosurgical Center, Lexington, KY.

Dr. Gilbert is a neurosurgeon, neuroimagist, pain \& addiction, medicine specialist, Spine and Brain Neurosurgical Center, Lexington, KY. Dr. Wheeler, Dr. Mick, and Dr. Herder are neurosurgeons, Spine and Brain Neurosurgical Center, Lexington, KY. Dr. Storey is a psychiatrist, Spine and Brain Neurosurgical Center, Lexington,

KY. Dr. Richardson is a physiatrist, Spine and Brain Neurosurgical Center, Lexington, KY. Dr. Watts is a neurologist, Spine and Brain Neurosurgical Center, Lexington, KY. Dr. Gyarteng-Dakwa, Dr. Marino and Dr. Siddiqi are anesthesiologists, pain medicine specialists, Spine and Brain Neurosurgical Center,

Lexington, KY. Dr. Kenney is a radiologist, neuroradiologist, Spine and Brain Neurosurgical Center, Lexington, KY. Ms. Broughton is a nurse practitioner, Spine and Brain Neurosurgical Center, Lexington, KY.

Address correspondence: John W. Gilbert, MD Spine \& Brain Neurosurgical Center Lexington, $\mathrm{KY}$ E-mail:

correspondence@sbncmd.com

Disclaimer: There was no external funding in the preparation of this manuscript. Conflict of interest: None.

Manuscript received: 09/01/2009 Revised manuscript received: 12/09/2009

Accepted for publication: $02 / 28 / 2009$

Free full manuscript: www.painphysicianjournal.com
Background: Because the symptoms of drug misuse are nonspecific and difficult to detect, pain physicians have relied heavily on the results of urine drug tests to diagnose and treat chronic noncancer pain in patients who are prescribed controlled substances. However, changes in Medicare local carrier determinations for Medicare Part B providers in Connecticut, Indiana, Kentucky, and New York went into effect on July 1, 2009, whereby qualitative drug screening was no longer recognized as medically reasonable and necessary in the treatment of patients with chronic noncancer pain unless the patient presents with suspected drug overdose.

Study Design: A retrospective review of urine drug testing services.

Objective: To determine the extent of urine drug testing in patients with chronic noncancer pain in a large, Kentucky neuroscience practice offering pain management services combined with neurologic and neurosurgical services to better understand the potential effects of recent changes to Medicare benefits.

Methods: An audit of services provided during 2007 was conducted using computer software.

Outcome Measures: Outcome measures included the number of practice services, number of urine drug tests by payor, and the number of noncompliant patients by payor who self-released from care.

Results: Urine drug tests represented approximately $18.2 \%$ of professional medical services rendered in 2007 to patients with a diagnosis of chronic noncancer pain. Of these, UDTs represented approximately $22.2 \%$ of services provided to Medicare patients and $24.6 \%$ of services provided to Medicaid patients. In 2007, 2,081 patients with noncompliant UDTs self released from the practice against medical advice. Of these, $23.1 \%$ were enrolled in Medicare and $47.5 \%$ were enrolled in Medicaid. Approximately $40 \%$ of patients were referred to the CARE Clinic on the basis of noncompliance as indicated by UDT and/ or behavioral health issues. Of these, approximately $50 \%$ remained in treatment. Urine drug tests were also instrumental in revealing that $19.6 \%$ of patients showed signs of drug abuse or addiction. Of these patients, approximately $60 \%$ were government insured.

Limitations: Not a prospective, double-blinded study. We approximated the proportion of patients potentially affected by drug abuse or addiction as the percentage of patients self releasing from medical care.

Conclusion: In 2007, UDTs were used as an effective tool in adherence monitoring in a private neuroscience practice in Kentucky that offers pain management services combined with neurologic and neurosurgical services. UDTs were instrumental in referring $40 \%$ of patients for evaluation and treatment by behavioral health and addiction medicine specialists. UDTs were also instrumental in discovering signs of drug abuse or addiction in $19.6 \%$ of patients. Of these patients, approximately $60 \%$ were government insured. Should the objective and reliable sign offered by UDTs be eliminated from the physician's toolbox, the physician's ability to accurately diagnose and treat these patients could be impaired.

Key words: Chronic noncancer pain, Medicare, Medicaid, urine drug testing, opioids, drug abuse

Pain Physician 2010; 13:187-194 
urrent literature describes common uses of prescription opioids in the treatment Jof chronic noncancer pain (1). However, the use of opioids carries considerable risk. In their comprehensive review of the literature, Manchikanti and Singh (2) found evidence that, in addition to common side effects such as nausea and sedation, long-term therapeutic use of opioids is associated with changes to the hormonal and immune system, abuse and addiction, tolerance, and hyperalgesia. They also found a considerable societal cost in increased disability, medical expenditures, and subsequent surgeries. Further, the effectiveness and appropriate use of opioids in chronic non-cancer pain continues to be controversial (2-7).

Because of these risks, the therapeutic use of opioids should be accompanied by a cohesive and coordinated regimen of adherence monitoring involving multiple instruments $(2,3,5,8-15)$. The specifics of adherence monitoring programs have been well described elsewhere (9-16). When knowledgeably used, urine drug testing has been shown to be a valuable, noninvasive tool to confirm compliance with controlled-substance use and/or to diagnose the misuse or abuse of controlled substances or the use of illicit drugs (8). For this reason urine drug testing has formed the cornerstone of adherence monitoring in controlled substance management of chronic pain. When knowledgeably interpreted, the urine drug test (UDT) offers the physician an objective, reliable sign of drug misuse and abuse unlike other nonspecific signs/symptoms (17).

Of the populations in which urine drug testing is commonly conducted, chronic noncancer pain patients are at highest risk for controlled substance misuse, both because of their medical need for therapeutic opioids and because of the common co-occurring medical conditions and other risk factors linked with chronic pain. Identifying potential drug misuse early enables the practitioner to refer patients, sooner rather than later, to specialists in behavioral health care and addiction medicine for potential treatment plan modifications. For such reasons, the cost of urine drug testing used in the treatment of chronic pain has traditionally been covered under many health insurance plans, including Medicare and Medicaid.

Point-of-care UDTs have been estimated to cost Medicare up to $\$ 220$ per UDT. In addition, when urine samples are sent to commercial laboratories these costs typically cost Medicare up to an additional $\$ 250$ per sample. Because these costs hold the potential for abuse by providers, they have been the subject of recent scrutiny by Medicare.

Further complicating cost considerations is the selection of UDT panels. There is no standard UDT panel that is suitable for all purposes and settings. Physicians should consider UDTs that are suitable for their clinical need, type of practice and clinic location. Typical UDT panels performed by commercial laboratories tend to test for a larger suite of drugs compared with UDTs designed for point-of-care. Commercial tests can include comprehensive profiles and additional metabolites and encompass substances such as amphetamine, amobarbital, butalbital, CIV alprazolam, carisoprodol, chlordiazepoxide, clonazepam, codeine, diazepam, dihydrocodeine, fentanyl, flurazepam, hydrocodone, hydromorphone, lorazepam, marijuana, meperidine, meprobamate, methamphetamine, methadone, 3,4methylenedioxymethamphetamine (MDMA or ecstasy), 3,4-methylenedioxyethamphetamine (eve), 3,4-methylenedioxyamphetamine (MDA), morphine, oxazepam, oxycodone, pentobarbital, propoxyphene, secobarbital, temazepam, as well as specimen validity testing utilizing creatinine, specific gravity, $\mathrm{pH}$, adulterant additives, and gas chromatography-mass spectrometry (GC-MS) confirmation when indicated by their own laboratory protocols.

In some states (18), including Kentucky, a new local carrier determination (LCD) was issued effective for dates of service on or after July 1, 2009, whereby Medicare now restricts reimbursement for routine qualitative drug screening in chronic pain patients. Under this LCD, a qualitative drug screen is medically reasonable and necessary for the monitoring of chronic pain patients in whom other illicit drug use is suspected. Unfortunately, the non-specific nature of most signs and symptoms of drug misuse now make it difficult for most physicians to identify early drug misuse without the aid of extensive questionnaires. Furthermore, the new LCD ignores chronic pain itself as a reason to do compliance testing, and rather requires signs and/or symptoms of potential drug misuse. Because many patients are unable to afford to pay for UDT laboratory panels out of pocket based on current Medicare fee schedules, many instances of drug misuse could thus go undetected. For example, patients who sell their medications may never show signs and/or symptoms of abuse. Thus, this change in Medicare policy holds the potential to reduce the standard of care for this complex patient population.

The impact of this coverage change has the potential to be disproportionately large in Kentucky. There, 
patients eligible for Medicare and Medicaid benefits have been shown to have high rates of abuse of illicit drugs, with Medicaid patients having a $60 \%$ rate of abuse and illicit drug use, and Medicare/Medicaid patients a $40 \%$ rate of abuse of illicit drugs (10). These numbers are consistent with statistics that show that comorbid medical conditions, low socioeconomic status, and tobacco smoking are linked with higher than average rates of prescription drug misuse and illicit drug use. For example, smoking, which is linked to a wide range of increased rates of cancer, heart disease, and other medical conditions is also linked to a 5 times higher than average rate of illicit drug use compared with nonsmoking (19). Unemployed adults were found to have a substance abuse rate $(18.3 \%)$ that was over twice that of those who were employed full time (8.4\%) (20).

We conducted a study to determine the extent of noncompliance among chronic noncancer pain patients who were insured by Medicaid and Medicare in our Kentucky neurosurgical and pain medicine practice. The goal of our study was to learn the extent to which UDT compliance testing is medically necessary in this patient population as a means of understanding the potential effects of these recent Medicare benefit changes.

\section{Methods}

\section{Study Design}

A retrospective study was conducted of urine drug testing services provided in 2007 by a large neurosurgical and pain management practice, which has 5 clinic locations in Central and Eastern Kentucky.

\section{Practice Description}

\section{Medical Staff}

Each of the 5 clinic locations is staffed with multispecialty physicians who either rotate among clinics in person or via telehealth technology. Practice physicians are board certified in specialties that include pain medicine, addiction medicine, behavioral health, psychiatry, physical medicine and rehabilitation, neurology, neuroimaging, neurosurgery, and anesthesiology. The medical staff also includes a behavioral health nurse practitioner, nurses, medical assistants, UDT technicians, and administrative personnel, employing between 50 to 70 people depending on clinic demand.

\section{Telehealth Technology}

The 5 clinics are connected by dedicated HIPAA- compliant T-lines to a central Citrix ${ }^{\circledR}$ service in the Lexington office via intranet using a 200-bit encryption and state-of-the-art firewalls that allow all telephone calls to be routed via voice over Internet protocol (VoIP) to a central call center. Medical staff can access patients' electronic medical records from examination rooms at any clinic location using real-time encrypted T-lines connected to the central servers in the Lexington facility, which run Impact.MD ${ }^{\mathrm{TM}}$ (Allscripts Co., Chicago, IL) electronic medical record software.

\section{Referrals}

The practice accepts patients primarily on a referral basis. Approximately 2,000 physicians have referred patients to this practice from a broad region spanning Central and Eastern Kentucky, Ohio, Tennessee, Virginia, and West Virginia. Referrals are made for a wide range of neuropathic pain diagnoses, the most common of which is chronic noncancer pain. Patients are referred for evaluation and management of pain, for neurosurgical evaluation and management, for injective therapies, imaging studies, intrathecal drug delivery with pump implants, spinal cord stimulators, and other procedures.

\section{CARE Clinic}

The practice provides an in-house CARE (Caring Approach to Redirect patient Expectations) clinic that is designed to re-educate, assess, and modify treatment paths of patients engaging in noncompliant behavior. The clinic is staffed by in-house behavioral health and addiction medicine specialists. With establishment of this clinic, the practice moved away from deterrence testing (random UDTs) to detection testing (routine and random UDTs). Detection testing is designed to improve diagnoses and to guide appropriate treatment or treatment modification. The rate of patient retention by the practice increased after the CARE clinic was established.

\section{Laboratory Certification}

The practice laboratory obtained a certificate of registration effective December 13, 2006, pursuant to section 353 under Public Health Services Act 42 USC 263(a) as revised by the Clinical Laboratory Improvement Amendments (CLIA), which demonstrates compliance with methods and assurances established by the CLIA. This certificate is required for billing Medicare and Medicaid for point-of-care UDT services. In Kentucky, CLIA regulations are administered through the 
Kentucky Cabinet for Health and Family Services, Office of Inspector General. Certificates are granted on the basis of periodic inspections of laboratory facilities and proficiency testing of UDT technicians and associated medical staff by the Inspector General's office.

\section{Patient Population}

The population was defined as patients with a chronic pain diagnosis (ICD code 338.29) who received urine drug testing services in 2007. Study inclusion and exclusion criteria are presented in Table 1. Of the 10,593 eligible patients, $53.2 \%(5,634)$ were female and $46.8 \%(4,959)$ were male. The mean age was 48.7 years. Of the total, $34.75 \%(3,681)$ were enrolled in Medicare and $31.0 \%(3,284)$ were enrolled in Medicaid. Over $80 \%$ of the patients were established and not new to this practice in 2007.

All patients provided written consent for urine drug testing and for publication of their medical information without personal identifiers. Appropriate precautions were taken and enforced to protect the privacy and identity of patients participating in the study.
The total number of patients receiving urine drug testing was determined by querying our billings database based on CPT code; patient population demographics were generated based on account number. Data analysis methods are described in more detail below.

\section{Medical History}

All patients referred for evaluation and pain management services underwent a comprehensive history including a 22-point compliance assessment and a review of a 45-paragraph consent form for treatment with or without opioids at each and every visit to reinforce and re-educate patients and family members regarding practice policies and procedures, compliance expectations, and signs of drug misuse. This precaution was followed because over $90 \%$ of patients referred to our practice were already taking opioids for their pain, and it is reported that about $90 \%$ of primary care physicians miss the signs and symptoms of drug abuse (21). Of those who were not taking opioids, most had since been considered for treatment with controlled substances.

Table 1. Patient inclusion and exclusion criteria.

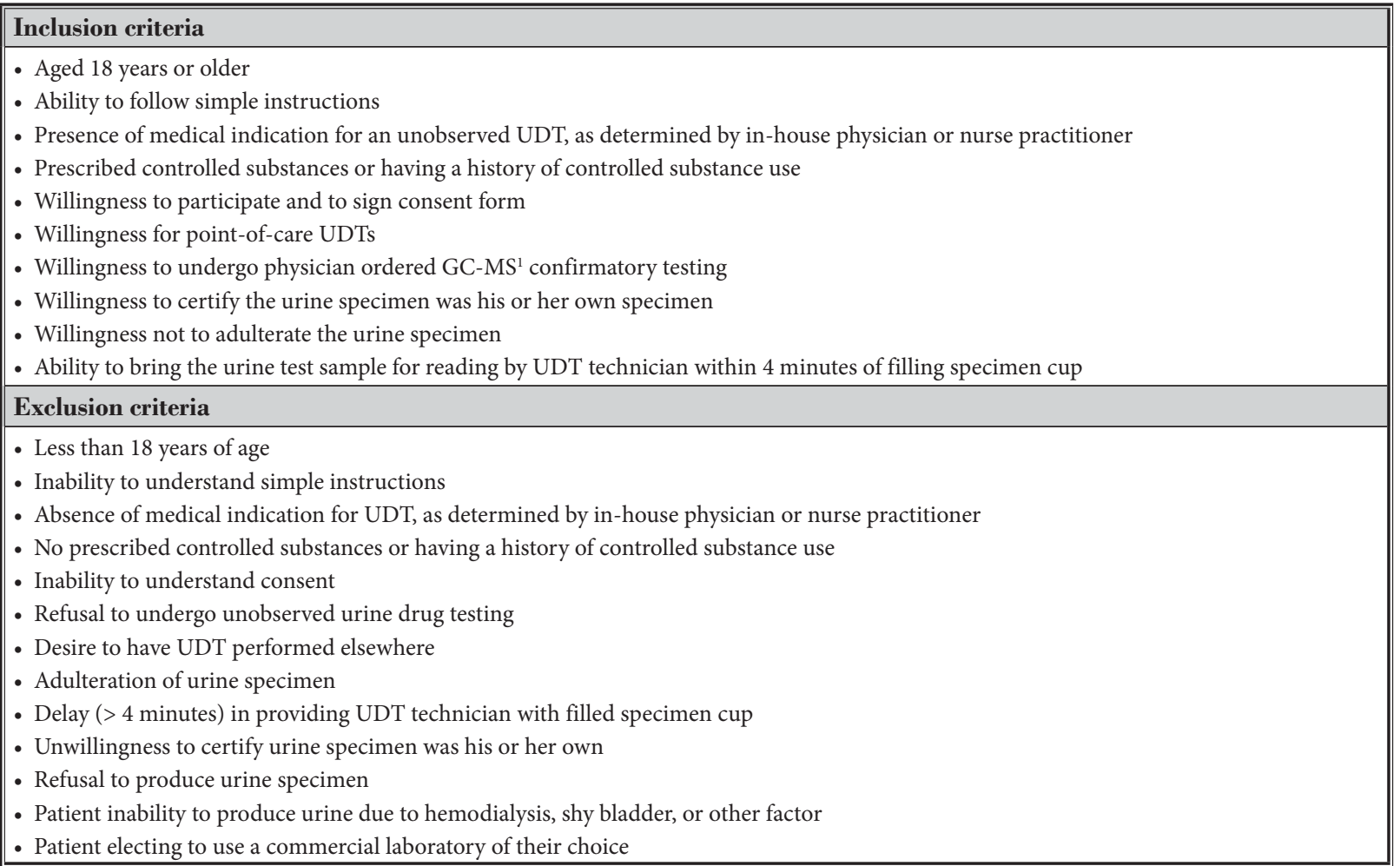

1 GC-MS - Gas chromatography mass spectrometry. 


\section{Urine Drug Testing}

Urine drug tests were administered to monitor patient compliance to opioid therapy for the treatment of chronic noncancer pain. Specifically, UDTs were used to diagnose compliance with a patient's treatment agreement; to detect, evaluate and diagnose noncompliance with the referring physician treatment plans; to evaluate patients being considered for surgery, injections, medications, or controlled substances including opioids; to establish a baseline measure for treatment; and to detect, evaluate and diagnose drug misuse, abuse or addiction (22-24). In our practice, we consider compliance (both random and routine) testing medically necessary for patients diagnosed with chronic pain; compliance testing was ordered by the treating physician.

Each patient who underwent urine drug testing did so to assist in diagnosis and treatment for the purpose of improving medical outcomes. This purpose for drug testing differs significantly from that of workplace drug testing, which is usually conducted at random intervals to deter, but not eliminate, drug use to improve employee work performance. Workplace drug testing expenses are paid by the employer and are not the subject of this study (25). Workplace drug testing is typically performed on individuals who have no signs or symptoms of drug misuse, whereas urine drug testing in this study was typically performed on patients who were symptomatic with pain and may have had signs/symptoms of drug misuse.

AdminiStar Federal, the local contractor for The Centers for Medicare and Medicaid Services (CMS), was contacted regarding proper procedures for documenting UDTs. No local coverage determination (LCD) was in place in 2007 and no UDT frequency limits were noted. Guidance was sought on the proper use and coding of UDTs. Typical neuroscience-related international classification of diseases (ICD) codes were recommended such as cervical, thoracic, or lumbar pain; headaches and certain ICD V-codes such as high-risk medication use. Additional documentation requirements such as physician orders, physician review of tests, and physician interpretation and certification of the medical necessity of UDTs were reviewed and verified with AdminiStar Federal. The practice had no prior issues with the local carrier or CMS related to urine drug testing (all patients previously went elsewhere). Insurers, including Medicare and Medicaid, were charged on a per UDT basis, rather than on a per drug basis (e.g., \$19 per UDT). All payors were billed the same rate for UDTs.

The urine drug tests were performed during patient office visits, utilizing the urine 12-panel drug im- munoassay iCup (Instant Technologies Inc., Norfolk, VA, USA). This product was chosen because it is designed as a rapid test for use by health care professionals at point of care and includes an adulteration strip that measures urine $\mathrm{pH}$, specific gravity, and oxidants such as bleach and hydrogen peroxide. In-house UDT technicians and medical staff were trained in implementing manufacturer and practice guidelines.

Test results were reviewed by the treating physician and integrated into the patient treatment plan. Patients were considered noncompliant if they tested positive for one or more illicit drugs, including cocaine, THC (tetrahydrocannabinol), amphetamine or methamphetamine, or non-prescribed benzodiazepine, opiates, or other medications. A positive test for cocaine was considered positive unless the patient could provide medical records from a dentist; ear, nose, and throat specialist; or other practitioner who verified the use of a procedure that had involved liquid cocaine within 72 hours before the UDT.

If the patient disagreed with the UDT result, confirmatory testing was performed by a commercial laboratory using GC/MS. The need for GC/MS testing and associated costs were greatly reduced, however, because all examination rooms were equipped with access to electronic medical records and on-line resources related to the interpretation of UDT results and because the medical staff was trained in patient interviewing techniques.

Patients were also considered noncompliant if the prescribed opioid did not show up in the UDT and its absence was confirmed by patient history or by GC/MS. UDTs were occasionally repeated at the request of the patient or the attending physician.

If patients were found to be noncompliant on UDT they were referred to the CARE clinic for evaluation by a behavioral health specialist and/or addiction medicine physician to determine whether drug misuse was experimental (e.g., youth), recreational (e.g., at a party), or circumstantial (e.g., chemical coping), or whether it constituted abuse or addiction. Referred patients were evaluated and treated for comorbid psychiatric conditions, as needed.

\section{Measurements}

\section{Extent of Urine Drug Testing}

The number of UDT services provided in 2007 to patients with chronic pain was determined with the use of Sage Practice Analytics software (Sage North America, 
Tampa, FL). All other practice services included evaluation and management codes (i.e., the CPT 9900 level codes) and services typically performed on unique dates of services such as pump refill or interrogation, stimulator interrogation or adjustment, interventional techniques, electroneurodiagnostics, magnetic resonance imaging services, facet rhizotomies, shunt interrogation adjustment, and CARE clinic. The percentage of UDTs was calculated as a percentage of all professional physician services and as a percentage of the services billed through Medicare or Medicaid. These percentages approximate the percentage of patient visits that involve urine drug testing.

\section{Measures of Potential Drug Abuse and Addiction}

The number of patients who declined treatment modifications and self released themselves from the practice against medical advice was selected as the primary, indirect measure of potential drug abuse and addiction. Possible treatment modifications included more frequent monitoring, reduction in or elimination of controlled substances, participation in a 12-step program, referral to a methadone clinic, behavioral modification counseling, alternative or adjunctive forms of treatment, vocational rehabilitation, spiritual counseling, and injective therapy.

\section{Results}

\section{Services}

The total number of urine drug tests (CPT code 80101) provided in 2007 was 30,946 (Table 2), which represents $18.2 \%$ of the 170,258 total services (procedures) rendered and billed to chronic pain patients in that year. Given a chronic pain patient population of 10,593 , the average number of urine drug tests that year was 3.4 UDTs per patient.

Medicare UDT services $(9,711)$ represented $5.7 \%$ of all practice services rendered and billed to all payors in 2007 . Urine drug tests represented $22.2 \%$ of all ser- vices provided to Medicare chronic pain patients. Similarly, UDTs represented $24.6 \%$ of all services provided to Medicaid chronic pain patients.

\section{GC/MS Confirmatory Testing}

The vast majority of patients who tested as noncompliant admitted to their incorrect initial history and most agreed to a modified treatment plan after conversation with their physician. Strong emphasis was placed on open communication between patients and physicians. The success of this approach is reflected in a consistently low rate (3-5\%) of requests by our physicians for GC/MS confirmatory testing.

\section{CARE Clinic Referrals}

Patients were referred to the CARE clinic based on UDT results and/or by physician request. Approximately $40 \%$ of patients were referred to the CARE Clinic on the basis of noncompliance as indicated by UDT and/or behavioral health issues. The vast majority of these referrals represent instances of experimental (e.g., youth), recreational (e.g., at a party), or circumstantial (e.g., chemical coping) use.

\section{Patient Retention}

In 2007, 2,081 patients with noncompliant UDTs released themselves from the practice against medical advice (Table 3). Of the patients who self released from care, $23.1 \%$ (481) were enrolled in Medicare and $37.5 \%$ (780) were enrolled in Medicaid. No other group of patients by payor exceeded $12 \%$ (data not shown). Twelve percent of patients covered by workers compensation carriers and less than $12 \%$ of patients covered by commercial payors self released from care.

\section{Discussion}

We assumed that noncompliant chronic pain patients self releasing from care represented those patients most likely to have the more severe diagnoses of

Table 2. Number of UDTs provided to Medicare and Medicaid chronic pain patients in a Kentucky private neurosurgical and pain management practice in 2007.

\begin{tabular}{|l|c|c|c|}
\hline Parameter & All payors & Medicare & Medicaid \\
\hline All services & 170,258 & $43,735(25.7 \%)$ & $50,404(29.6 \%)$ \\
\hline UDTs, $n$ & 30,946 & 9,711 & 12,417 \\
\hline UDTs, \% of services by payor & 18.2 & 22.2 & 24.6 \\
\hline
\end{tabular}


drug abuse or addiction. These included patients who declined treatment modifications and/or were unable to complete recommendations from the psychiatrist and/or addiction physician. In most cases, these patients were not ready to change their behavior and were not receptive of nonopiate pain management. They were given referral information to 12-step programs, methadone clinics, and other resources. In 2007, patients self releasing from care represented $19.6 \%$ of all chronic pain patients. In comparison, approximately $60 \%$ of those who self released from care were insured by Medicare or Medicaid.

Manchikanti et al (9), in their study of the efficacy of adherence monitoring in their pain management practice in western Kentucky, reported a drug abuse rate of $17.8 \%$ as indicated by doctor shopping and trafficking. Although the indicators being measured differ between our study and that of Manchikanti et al, the drug abuse rate appears to be similar despite presumed differences in patient demographics between the 2 practices. First, our practice provides pain management combined with neurologic and neurosurgical services. Second, Manchikanti et al's practice is located in the western third of the state, whereas our referral area includes the Appalachian region; drugs of abuse tend to vary regionally.

It is important to note that the majority of patients accepted the provision of in-house treatment plan modifications in response to noncompliant UDT results and remained patients in the practice. The primary purpose of UDTs was to assist physicians in diagnosing drug misuse early. Of the $40 \%$ of patients who were referred to our CARE clinic for evaluation and treatment, approximately $50 \%$ remained in treatment. Noncompliance issues among the majority of these patients involved drug misuse primarily in the forms of experimental (e.g., youthful), recreational (e.g., social settings), or circumstantial (e.g., chemical coping) use. Noncompliance also includes use of illicit substances other than the prescribed drug.

Otherwise, the rate of urine drug testing among Medicare $(22.2 \%)$ and Medicaid $(24.6 \%)$ patients was slightly higher than that of the chronic noncancer pain population of the practice as a whole (18.2\%). However, these elevated numbers lag behind the percent of total services rendered to Medicare $(25.7 \%)$ and Medicaid $(29.6 \%)$ chronic pain patients.

The early stages of drug misuse are difficult to detect without urine drug testing. In over $90 \%$ of patients who misuse drugs on an infrequent or occasional basis,
Table 3. Noncompliant chronic pain patients self releasing from care in 2007.

\begin{tabular}{|l|c|c||}
\hline Payor & $\begin{array}{c}\text { Patients } \\
\text { (n) }\end{array}$ & $\begin{array}{c}\text { Of patients self- } \\
\text { releasing } \\
(\%)\end{array}$ \\
\hline All payors & 2,081 & 100.0 \\
\hline Medicare & 481 & 23.1 \\
\hline Medicaid & 780 & 37.5 \\
\hline
\end{tabular}

our medical staff had already noted on charts "no diagnosis of abuse/addiction." Urine drug testing allows the patient to be counseled about the significance of a noncompliance diagnosis and about recreational or circumstantial or experimental drug use. Treatment contracts can be reviewed and treatment modifications can be made (e.g., more frequent monitoring, decreased opioids, use of nonopioids, increased adjunctive measures, behavioral modification/counseling, education).

The national cost of opioid abuse is enormous and cited as ranging from as high as $\$ 300$ billion annually as per estimates of the White House Budget Office, including the costs of crime, health care, accidents, and lost productivity (4). Our experience has shown improved outcomes and treatment retention by diagnosing drug misuse at the earlier stages of experimental, recreational, or circumstantial use compared with cases in which drug misuse or illicit substance use has progressed to the point of addiction. Detection of drug misuse can aid in identifying patients not suited for opiate-based chronic pain management.

\section{Conclusion}

The primary purpose of UDTs is to assist physicians in diagnosing drug misuse early. Urine drug tests used in adherence monitoring in a private neuroscience practice in Kentucky offering pain management services combined with neurologic and neurosurgical services were instrumental in referring $40 \%$ of patients for evaluation and treatment by behavioral health and addiction medicine specialists. Of these, approximately $50 \%$ remained in treatment. Urine drug tests were also instrumental in discovering signs of drug abuse or addiction in $19.6 \%$ of patients. Of these patients, approximately $60 \%$ were government insured.

Physicians rely heavily on the results of UDTs in diagnosing and treating chronic pain patients with controlled substances, because the symptoms of drug misuse are non-specific and difficult to detect (e.g., ner- 
vousness, anxiety, depression, change in sleep pattern or appetite, change in behavior, yawning, diarrhea, nausea, age less than 35 years or elderly, alcohol consumption, muscle aches, and numbness) (17). Should the objective and reliable sign offered by UDT results be eliminated from the physician's toolbox, the physician's ability to accurately diagnose and treat these patients could be impaired. Impaired ability to serve a patient population at high risk for substance abuse could potentially lead to poorer patient outcomes and possibly jeopardize recent strides to combat prescription drug abuse, especially in states with large high-risk populations like Kentucky.

\section{References}

1. Ballantyne J. Opioid analgesia: Perspectives on right use and utility. Pain Physician 2007; 10:479-481.

2. Manchikanti L, Singh A. Therapeutic opioids: A ten-year perspective on the complexities and complications of escalating use, abuse, and nonmedical use of opioids. Pain Physician 2008; 11:S63-S88.

3. Ballantyne J, Mao J. Opioid therapy for chronic pain. New Engl J Med 2003; 349:1943-1953.

4. Trescot AM, Helm S, Hansen $\mathrm{H}, \mathrm{Be}$ nyamin R, Glaser S, Adlaka R, Patel S, Manchikanti L. Opioids in the management of chronic non-cancer pain: An update of the American Society of Interventional Pain Physicians' (ASIPP) guidelines. Pain Physician 2008; 11:S5-S62.

5. Smith H. Peripherally-Acting Opioids. Pain Physician 2008; 11:S121-S132.

6. Trescot AM, Datta S, Lee M, Hansen H. Opioid Pharmacology. Pain Physician 2008; 11:S133-S153.

7. Trescot AM, Glaser SE, Hansen H, Benyamin RM, Patel S, Manchikanti L. Effectiveness of Opioids in the treatment of chronic non-cancer pain. Pain Physician 2008; 11:S181-S200.

8. Manchikanti L, Atluri S, Trescot AM, Giordano J. Monitoring opioid adherence in chronic pain patients: Tools, techniques, and utility. Pain Physician 2008; 11:S155-S180.

9. Manchikanti L, Manchukonda R, Damron K, Brandon D, McManus C, Cash $\mathrm{K}$. Does adherence monitoring reduce controlled substance abuse in chronic pain patients? Pain Physician 2006; 9:57-60.

10. Manchikanti L, Fellows B, Damron KS, Pampati V, McManus CD. Prevalence of illicit drug use among individuals with chronic pain in the Commonwealth of Kentucky: an evaluation of patterns and trends. J Ky Med Assoc 2005 Feb; 103(2):55-62.

11. Manchikanti KN, Manchikanti L, Damron KS, Pampati V, Fellows B. Increasing deaths from opioid analgesics in the United States: An evaluation in an interventional pain management practice. J Opioid Manage 2008; 4:271-283.

12. Manchikanti L, Giordano J, Boswell MV, Fellows B, Manchukonda R, Pampati V. Psychological factors as predictors of opioid abuse and illicit drug use in chronic pain patients. J Opioid Manage 2007; 3:89-100.

13. Manchikanti L, Benyamin RM. Longterm opioid therapy. Pain Pract 2009; 9:164.

14. Manchikanti L, Manchikanti KN, Pampati V, Cash KA. Prevalence of side effects of prolonged low or moderate dose opioid therapy with concomitant benzodiazepine and/or antidepressant therapy in chronic non-cancer pain. Pain Physician 2009; 12:259-267.

15. Manchikanti L, Pampati V, Damron KS, McManus CD. Evaluation of variables in illicit drug use: Does a controlled substance abuse screening tool identify illicit drug use? Pain Physician 2004; 7:71-75.

16. Gilbert JW, Wheeler GR, Lingreen RA, Martonffy D, Hatchett J, Gaines R, Stidham S, Sutton T, Westerfield G, McConnell M, Noble S, Carter K. The ten Cs of chronic noncancer pain: Universal precautions for the chronic noncancer pain patient. Am J Pain Manage 2005; 15:2232.

17. Narconon International. Signs and symptoms of drug use.

www.narconon.org/parent_center/ signs_symptoms. Accessed: May 29, 2009

18. Centers for Medicare and Medicaid Services. Qualitative drug screening L28145.

www.ngsmedicare.com/NGSMedicare/lcd/DL28145_f_lcd.htm.

19. U.S. Department of Health and Human Services. Summary of findings from the 2000 national household survey on drug abuse. In: Substance Abuse and Mental Health Services Administration, editor.; 2000.

20. Buck J, Miller K. Mental health and substance abuse services in Medicaid, 1995. DHHS Pub. No. (SMA) 023713. Rockville, MD: Center for Mental Health Services, Substance Abuse and Mental Health Services Administration, 2002.

21. Goldfarb B. Primary care physicians often miss signs of drug abuse in pain patients. Pain Med News 2005; 3:6.

22. Kentucky Board of Medical Licensure: Model guidelines for the use of controlled substances in pain treatment. J Ky Med Assoc 2001; 99:291-294.

23. Campbell A. American Academy of Pain Management Pain Program Accreditation: Description and value. www.sbncmd.com/Pain\%20Managem ent\%20Program\%20Accreditation.pdf. Accessed June 25, 2009.

24. Kozma A. Urine drug screening in everyday practice. Practical Pain Management 2007(April):18-20.

25. Evans M, Ma'Ayteh B, Constantine J. Guest editorial. Practical Pain Management 2005 (Jul/Aug). 